Draft version October 1, 2018

Typeset using LATEX twocolumn style in AASTeX61

\title{
ON THE DISAPPEARANCE OF A COLD MOLECULAR TORUS AROUND THE LOW-LUMINOSITY ACTIVE GALACTIC NUCLEUS OF NGC 1097
}

T. Izumi,${ }^{1}$ K. Kohno,${ }^{2,3}$ K. Fathi,${ }^{4}$ E. Hatziminaoglou,${ }^{5}$ R. I. Davies,${ }^{6}$ S. Martín $,{ }^{7}, 8$ S. Matsushita,${ }^{9}$ E. Schinnerer,${ }^{10}$ D. Espada, ${ }^{1,11}$ S. Aalto, ${ }^{12}$ K. Onishi,${ }^{13}$ J. L. Turner,${ }^{14}$ M. Imanishi $,{ }^{1,11}, 15$ K. Nakanishi,${ }^{1,11}$ D. S. Meier, ${ }^{16}$ K. Wada, ${ }^{17}$ N. KaWaKatu, ${ }^{18}$ and T. NaKajima ${ }^{19}$

\footnotetext{
${ }^{1}$ National Astronomical Observatory of Japan, 2-21-1 Osawa, Mitaka, Tokyo 181-8588, Japan

${ }^{2}$ Institute of Astronomy, Graduate School of Science, The University of Tokyo, 2-21-1 Osawa, Mitaka, Tokyo 181-0015, Japan

${ }^{3}$ Research Center for the Early Universe, Graduate School of Science, The University of Tokyo, 7-3-1 Hongo, Bunkyo, Tokyo 113-0033, Japan

${ }^{4}$ Valhallav. 67, 11428 Stockholm, Sweden

${ }^{5}$ European Southern Observatory, Karl-Schwarzschild-Str. 2, 85748 Garching bei München, Germany

${ }^{6}$ Max-Planck-Institute für Extraterrestrische Physik, Postfach 1312, D-85741 Garching, Germany

${ }^{7}$ Joint ALMA Observatory, Alonso de Córdova 3107, Vitacura, Santiago 763-0355, Chile

${ }^{8}$ European Southern Observatory, Alonso de Córdova 3107, Vitacura, Santiago 763-0355, Chile

${ }^{9}$ Academia Sinica, Institute of Astronomy \& Astrophysics, P.O. Box 23-141, Taipei 10617, Taiwan

${ }^{10}$ Max Planck Institute for Astronomy, Königstuhl 17, Heidelberg 69117, Germany

${ }^{11}$ SOKENDAI (The Graduate University for Advanced Studies), 2-21-1 Osawa, Mitaka, Tokyo 181-8588, Japan

${ }^{12}$ Department of Space, Earth and Environment, Chalmers University of Technology, Onsala Observatory, SE-439 92 Onsala, Sweden

${ }^{13}$ Research Center for Space and Cosmic Evolution, Ehime University, 2-5 Bunkyocho, Matsuyama, Ehime 790-0826, Japan

${ }^{14}$ Department of Physics and Astronomy, UCLA, 430 Portola Plaza, Los Angeles, CA 90095-1547, USA

${ }^{15}$ Subaru Telescope, NAOJ, 650 North Aohoku Place, Hilo, HI 96720, USA

${ }^{16}$ Department of Physics, New Mexico Institute of Mining and Technology, 801 Leroy Place, Soccoro, NM 87801, USA

${ }^{17}$ Kagoshima University, Kagoshima 890-0065, Japan

${ }^{18}$ Faculty of Natural Sciences, National Institute of Technology, Kure College, 2-2-11 Agaminami, Kure, Hiroshima 737-8506, Japan

${ }^{19}$ Institute for Space-Earth Environmental Research, Nagoya University, Furo-cho, Chikusa-ku, Nagoya, Aichi 464-8601, Japan
}

(Received June 22, 2017; Accepted July 18, 2017)

\begin{abstract}
We used the Atacama Large Millimeter/submillimeter Array (ALMA) to map the $\mathrm{CO}(3-2)$ and the underlying continuum emissions around the type 1 low-luminosity active galactic nucleus (LLAGN; bolometric luminosity $\lesssim 10^{42}$ $\mathrm{erg} \mathrm{s}^{-1}$ ) of NGC 1097 at $\sim 10 \mathrm{pc}$ resolution. These observations revealed a detailed cold gas distribution within a $\sim 100$ pc of this LLAGN. In contrast to the luminous Seyfert galaxy NGC 1068, where a $\sim 7$ pc cold molecular torus was recently revealed, a distinctively dense and compact torus is missing in our $\mathrm{CO}(3-2)$ integrated intensity map of NGC 1097. Based on the $\mathrm{CO}(3-2)$ flux, the gas mass of the torus of NGC 1097 would be a factor of $\gtrsim 2-3$ less than that found for NGC 1068 by using the same CO-to- $\mathrm{H}_{2}$ conversion factor, which implies less active nuclear star formation and/or inflows in NGC 1097. Our dynamical modeling of the $\mathrm{CO}(3-2)$ velocity field implies that the cold molecular gas is concentrated in a thin layer as compared to the hot gas traced by the $2.12 \mu \mathrm{m} \mathrm{H}_{2}$ emission in and around the torus. Furthermore, we suggest that NGC 1097 hosts a geometrically thinner torus than NGC 1068. Although the physical origin of the torus thickness remains unclear, our observations support a theoretical prediction that geometrically thick tori with high opacity will become deficient as AGNs evolve from luminous Seyferts to LLAGNs.
\end{abstract}

Keywords: galaxies: active — galaxies: Seyfert — galaxies: individual (NGC 1097) — galaxies: ISM

Corresponding author: Takuma Izumi

takuma.izumi@nao.ac.jp 


\section{INTRODUCTION}

The unified scheme of active galactic nuclei (AGNs) postulates that the existence (type-1) or absence (type2) of a broad line region depends on the viewing angle of an optically and geometrically thick dusty/molecular torus (Antonucci 1993). Spatially resolved thermal dust emissions at near- to mid-infrared (NIR to MIR) wavelengths in AGNs support the existence of compact (< 10 pc) obscuring structures (e.g., Jafe et al. 2004; Burtscher et al. 2013).

In addition to the photometric evidence, Hicks et al. (2009) unveiled 10 pc scale molecular disks with high velocity dispersions through dynamical modeling of the $2.12 \mu \mathrm{m} \mathrm{H} \mathrm{H}_{2}$ (hereafter $\mathrm{NIR} \mathrm{H}_{2}$ ) emission line in local AGNs, which would correspond to the outer envelope of tori. These high velocity dispersion regions were also observed in NGC 1068 (Imanishi et al. 2016; García-Burillo et al. 2016; Gallimore et al. 2016), NGC 1377 (Aalto et al. 2017), and Centaurus A (Cen A; Espada et al. 2017) with the Atacama Large Millimeter/submillimeter Array (ALMA), in cold gas and dust emission. However, the physical origin of the high velocity dispersion or the vertical height of a torus is still being debated.

Some models suggest that a torus is an evolving structure energetically driven by, for example, massive inflows or a nuclear starburst (e.g., Vollmer et al. 2008; Wada et al. 2009), both of which can ignite the AGN activity. If those triggers become weak, the geometric thickness of the torus cannot be sustained. Disk winds (Elitzur \& Shlosman 2006; Nomura et al. 2016) or outflows driven by the AGN radiation (Wada 2012) themselves will also generate a toroidal structure. In either case, the geometry and opacity of the torus would depend on the AGN luminosity. This prompted us to investigate low-luminosity AGNs (LLAGN, bolometric luminosity $L_{\text {bol }} \lesssim 10^{42}$ erg s${ }^{-1}$; Ho 2008), where thick tori may be deficient due to insufficient energy input. Indeed, theories predict such deficiency at this LLAGN regime (e.g., Elitzur \& Shlosman 2006). Recent clumpy torus modelings of infrared spectral energy distributions (González-Martín et al. 2015, 2017), as well as kinematic modelings of the $\mathrm{NIR} \mathrm{H}_{2}$ emission line (Müller-Sánchez et al. 2013), of AGNs with various $L_{\text {bol }}$ supported the trend that LLAGNs have geometrically and optically thinner tori than luminous Seyferts.

Here, we present our 10 pc diameter resolution ALMA cycle 3 observations of $\mathrm{CO}(3-2)\left(\nu_{\text {rest }}=345.796 \mathrm{GHz}\right)$ and the underlying continuum emissions toward the central $\sim 100 \mathrm{pc}$ circumnuclear disk (CND) region of the nearby type-1 (Storchi-Bergmann et al. 1993) LLAGN of NGC 1097 ( $D=14.5 \mathrm{Mpc}, 1^{\prime \prime}=70$ pc; Tully 1988).
Considering its low-luminosity $\left(L_{\mathrm{bol}}=8.6 \times 10^{41} \mathrm{erg}\right.$ $\mathrm{s}^{-1}$; Nemmen et al. 2006), a comparison of observed torus properties of this LLAGN with those of luminous Seyferts such as NGC $1068\left(L_{\text {bol }}=2 \times 10^{45} \mathrm{erg} \mathrm{s}^{-1}\right.$; Marinucci et al. 2016) will shed light on the torus evolution. Given that the NIR $\mathrm{H}_{2}$ emission has complex excitation mechanisms and may reflect a small fraction of the molecular mass, cold gas observations could be beneficial for this kind of comparison. Note that the dynamical mass $\left(M_{\mathrm{dyn}}\right)$ at $r<30 \mathrm{pc}$ is $\times 2.5$ higher in NGC 1068 than in NGC 1097 (Hicks et al. 2009). Furthermore, based on high resolution ALMA cycle 1 data, Hatziminaoglou et al. (in prep.) found that the black hole mass $\left(M_{\mathrm{BH}}\right)$ of NGC 1097 to be smaller than that $\left(\sim 10^{7} M_{\odot}\right.$; Greenhill et al. 1996) of NGC 1068. Therefore, if the torus thickness is solely determined by the disk gravity, we would expect a thinner torus in NGC 1068 than in NGC 1097, which contrasts with the evolutionary torus models.

\section{OBSERVATIONS}

Our ALMA cycle 3 observations consisted of four executions using the band 7 receiver (ID \#2015.1.00126.S) with 38-39 antennas in September 2016, with a total onsource time of $2.8 \mathrm{~h}$. One of the four spectral windows (each with a width of $1.875 \mathrm{GHz}$ ) was used to fully cover the $\mathrm{CO}(3-2)$ emission line. The projected baselines ranged from $15.1 \mathrm{~m}$ to $3.1 \mathrm{~km}$, resulting in a maximum recoverable scale of $\sim 7^{\prime \prime}$. The reduction and calibration were conducted with CASA version 4.7 (McMullin et al. $2007)$ in the standard manner. To improve the image fidelity of the $\mathrm{CO}(3-2)$ cube, we combined cycle 3 and cycle 1 visibilities (ID \#2012.1.00187.S). The baseline of the cycle 1 data (three executions) ranged from 15.1 $\mathrm{m}$ to $1.6 \mathrm{~km}$ with $33-49$ antennas. The total on-source time of the combined cube is $4.0 \mathrm{~h}$. Continuum emission was subtracted individually from each execution data in the $u v$-plane.

All images presented in this paper were reconstructed using the CLEAN task with Briggs weighting (robust = 0.5). The resultant synthesized beams were $0^{\prime \prime} .17 \times$ $0^{\prime \prime} .11$ (P.A. $\left.=88^{\circ} .8\right)$ for the combined $\mathrm{CO}(3-2)$ cube, $\sim 0^{\prime \prime} .1$ for the cycle 3 continuum maps $\left(\nu_{\text {rest }}=350.3\right.$ $\mathrm{GHz})$, and $\sim 0^{\prime \prime} .2-0^{\prime \prime} .25$ for the cycle 1 continuum maps, which correspond to $\sim 7-15 \mathrm{pc}$. The $1 \sigma$ sensitivities were $0.84 \mathrm{mJy}^{\text {beam }^{-1}}$ for the combined $\mathrm{CO}(3-$ 2) cube (velocity resolution $d V=10 \mathrm{~km} \mathrm{~s}^{-1}$ ), 45-72 $\mu \mathrm{Jy}_{\text {beam }}{ }^{-1}$ for the cycle 3 continuum maps, and 70 $110 \mu \mathrm{Jy} \mathrm{beam}^{-1}$ for the cycle 1 continuum maps. The adopted absolute flux uncertainty was 10\% according to the ALMA Cycle 3 Proposer's Guide, which is included in our quantitative results. Although we lost 
$\sim 75 \%$ of the $\mathrm{CO}(3-2)$ flux as compared to the $18^{\prime \prime}$ aperture Atacama Pathfinder EXperiment (APEX) telescope (Piñol-Ferrer et al. 2011), this would have had little impact on our study as we focused on the central $1^{\prime \prime}$. Our combined cubes were further analyzed with MIRIAD (Sault et al. 1995).

\section{RESULT}

\subsection{Continuum emission}

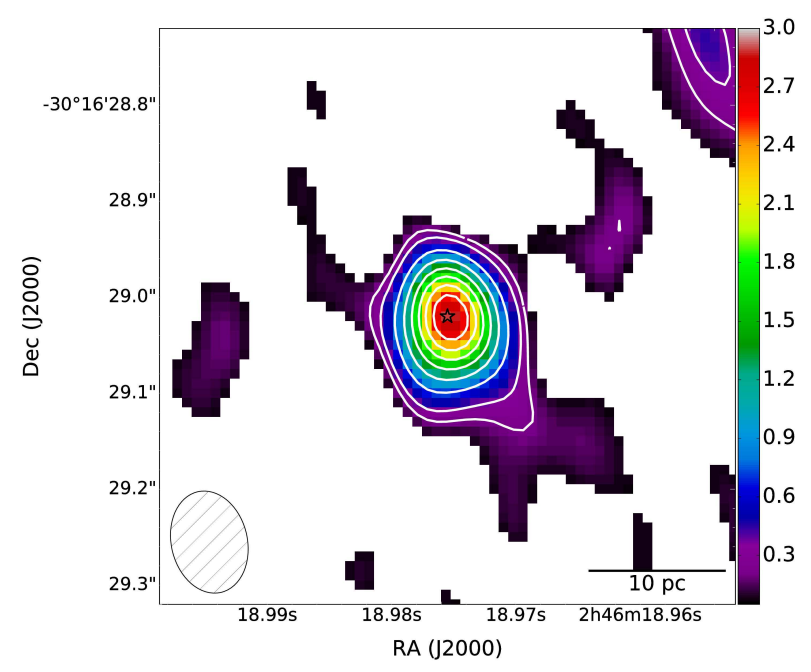

Figure 1. Band $7\left(\nu_{\text {rest }}=350.3 \mathrm{GHz}\right)$ continuum emission at the central region of NGC 1097, shown in both the color scale (mJy beam ${ }^{-1}$ unit) and contours $(3,5,10,15, \ldots, 40 \sigma$, where $1 \sigma=72.2 \mu \mathrm{Jy}$ beam $\left.^{-1}\right)$. This map is made with the data obtained on September 14, 2016, as the brightest example of the seven continuum measurements at different dates (see also Figure 2). The bottom-left ellipse indicates the synthesized beam $\left(0^{\prime \prime} .11 \times 0^{\prime \prime} .08\right.$, P.A. $\left.=13^{\circ} .0\right)$. The star marks the AGN position.

Figure 1 shows the spatial distribution of the ALMA band 7 continuum emission at the central $\sim 40 \mathrm{pc}$ of NGC 1097. This is made from the one cycle 3 execution data (14 September, 2016; $0^{\prime \prime} .11 \times 0^{\prime \prime} .08$ resolution). The $350 \mathrm{GHz}$ continuum emission peaks at $\alpha_{\mathrm{J} 2000.0}=$ $02^{\mathrm{h}} 46^{\mathrm{m}} 18^{\mathrm{s}} .9755, \delta_{\mathrm{J} 2000.0}=-30^{\circ} 16^{\prime} 29^{\prime \prime} .02$, which coincides precisely with the $14.9 \mathrm{GHz}$ peak position identified with a $1^{\prime \prime} .15 \times 0^{\prime \prime} .45$ beam (Orienti \& Prieto 2010). Thus we define this as the AGN location.

A Gaussian fit to the visibilities with the MIRIAD task uvfit revealed that this emission was unresolved at our $\sim 7 \mathrm{pc}$ resolution. This is in contrast to the case of NGC 1068, in which a dusty torus with a $\sim 7-10 \mathrm{pc}$ extent (diameter) was identified at 266 $\mathrm{GHz}$ and $694 \mathrm{GHz}$ continuum emission (Imanishi et al. 2016; García-Burillo et al. 2016; Gallimore et al. 2016). A dusty torus in NGC 1097 must have a very low surface brightness at $350 \mathrm{GHz}$ or be compact compared with the beam size.

We found significant time variability of the $350 \mathrm{GHz}$ continuum flux level in the central $r \lesssim 9$ pc during our seven executions (Figure 2). This is genuine as (i) the variability amplitude (factor $\sim 2-5$ around the mean $=1.3 \mathrm{mJy}$ ) was well beyond the absolute flux uncertainty and (ii) the simultaneously observed $\mathrm{CO}(3-2)$ flux remained almost constant over the executions. Difference in apertures between cycles 1 and 3 does not influence the result as the continuum emission is unresolved. Therefore, the band 7 continuum is dominated by this time-varying component, which seems to be the nonthermal synchrotron emission of a radio jet according to the spectral energy distribution modeling by Hatziminaoglou et al. (in prep.).

An upper limit on thermal dust emission then comes from our lowest continuum flux, i.e., $0.25 \pm 0.08 \mathrm{mJy}$. Following the method in $\S 3$ of García-Burillo et al. (2016), we estimated a mass of corresponding cold dust $M_{\text {dust }}<530 \pm 30 M_{\odot}$ within a single beam $\left(0^{\prime \prime} .26\right.$ $\left.\times 0^{\prime \prime} .13\right)$ of that observation, where we assumed a modified black body spectrum with an emissivity in$\operatorname{dex} \beta=2$ and a dust emissivity $\kappa_{352 \mathrm{GHz}}=0.0865$ $\mathrm{m}^{2} \mathrm{~kg}^{-1}$ (Klaas et al. 2001). A dust temperature of $150 \mathrm{~K}$ was assumed, which is a representative molecular gas kinetic temperature at the central $r<120 \mathrm{pc}$ (Izumi et al. 2013). If we decrease the dust temperature by $50 \%, M_{\text {dust }}$ is accordingly increased by $\sim 50 \%$ as it is close to the Rayleigh-Jeans regime. The time-variable nature clearly indicates that one should avoid using a single continuum measurement at the band 7 (and lower frequency) to estimate $M_{\text {dust }}$.

\section{2. $C O(3-2)$ emission}

Figure $3 \mathrm{a}$ shows the global distribution of the $\mathrm{CO}(3-$ 2 ) integrated intensity in the central $\sim 500 \mathrm{pc}$. The region mainly consists of (i) two spiral arms (labeled 1 and 3 in Figure 3b) that would connect to the kpc radius starburst ring (e.g., Quillen et al. 1995) and converge to (ii) the CND $(r \lesssim 100 \mathrm{pc}$ region; Izumi et al. 2013) that hosts the putative dusty/molecular torus. In addition to the two strong arms, there may be another two weak arms (labeled 2 and 4 in Figure 3b, see also van de Ven \& Fathi 2010). Although most of these structures have been identified in optical to NIR observations (Lou et al. 2001; Prieto et al. 2005; Fathi et al. 2006; Davies et al. 2009; van de Ven \& Fathi 2010; Fathi et al. 2013), we map them at cold gas emission that would reflect the bulk of the gas mass here.

Figure $3 \mathrm{c}$ shows a close-up view of the CND probed by $\mathrm{CO}(3-2)$ emission. The spiral arms jointly form an arc- 


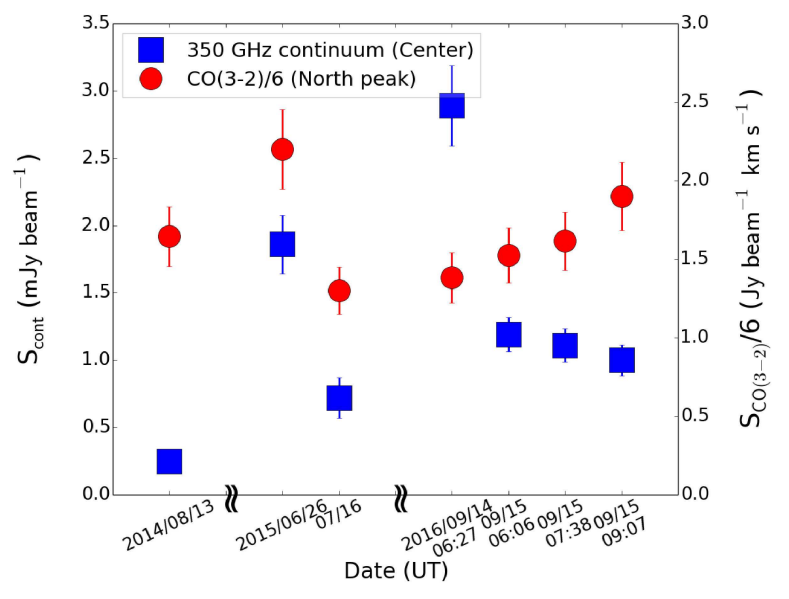

Figure 2. Time variation of the $\nu_{\text {rest }}=350.3 \mathrm{GHz}$ continuum emission at the central $r \lesssim 9$ pc of NGC 1097. The horizontal axis indicates the starting date of each execution. The $\mathrm{CO}(3-2)$ integrated intensities (matched $u v$ range: 40 to $1480 \mathrm{k} \lambda$; matched resolution $\left.=0^{\prime \prime} .3\right)$ measured at the northknot (Figure $3 \mathrm{c}$ ) are shown as the references.

like structure with two (north and south) bright knots. This arc may correspond to the nuclear star forming region inferred by Storchi-Bergmann et al. (2005). We also found $\mathrm{a} \sim 10 \mathrm{pc}$ spatial offset of the AGN position from the arc. Interestingly, a distinctive and compact molecular torus is missing at the AGN position of NGC 1097 in this CO(3-2) map. This is not due to too high gas excitation conditions at the center as we find a similar distribution in an $\mathrm{HCN}(4-3)$ integrated intensity map as well, which traces a factor of $\sim 1000$ denser gas (Kohno et al. in prep.).

\section{DISCUSSION ON THE TORUS AND SUMMARY}

To discuss torus properties in NGC 1097, we first estimate $\mathrm{H}_{2}$ mass $\left(M_{\mathrm{H}_{2}}\right)$ and the corresponding $\mathrm{H}_{2}$ column density $\left(N_{\mathrm{H}_{2}}\right)$ averaged within a single beam $\left(0^{\prime \prime} .17\right.$ $\times 0^{\prime \prime} .14$, P.A. $\left.=88^{\circ} .8\right)$ placed at the AGN position. The $\mathrm{CO}(3-2)$ integrated intensity at this position, 2.04 $\pm 0.21 \mathrm{Jy} \mathrm{beam}^{-1} \mathrm{~km} \mathrm{~s}^{-1}$, was converted to that of $\mathrm{CO}(1-0)$ by using a $\mathrm{CO}(3-2) / \mathrm{CO}(1-0)$ brightness temperature ratio of $2.33 \pm 0.44$ obtained at the central $7^{\prime \prime} .9 \times 3^{\prime \prime} .2$ aperture of NGC 1097 (Hsieh et al. 2012). By assuming a CO-to- $\mathrm{H}_{2}$ conversion factor $\left(X_{\mathrm{CO}}\right)$ of 0.5 $\times 10^{20} \mathrm{~cm}^{-2}\left(\mathrm{~K} \mathrm{~km} \mathrm{~s}^{-1}\right)^{-1}$ (García-Burillo et al. 2014), we obtain ${ }^{1} M_{\mathrm{H}_{2}}=(5.4 \pm 0.4) \times 10^{4} M_{\odot}$ and $N_{\mathrm{H}_{2}}=$ $(1.9 \pm 0.4) \times 10^{22} \mathrm{~cm}^{-2}$. This $N_{\mathrm{H}_{2}}$ is $\sim 100$ times larger than that derived from the X-ray spectral analysis (Nemmen et al. 2006), but it can be a natural consequence of the type-1 (face-on) torus geometry. As the

${ }^{1} \mathrm{~A} \sim 0.3$ dex uncertainty of this $X_{\mathrm{CO}}$ is not considered here.
$M_{\mathrm{H}_{2}}$ derived with this $X_{\mathrm{CO}}$ and that from thermal dust emission agrees fairy well for the molecular torus of NGC 1068 (García-Burillo et al. 2014, 2016), our use of this factor would be a good starting point for comparisons with NGC 1068. This $X_{\mathrm{CO}}$ is also comparable to that recommended for active environments by Bolatto et al. (2013). However, it will be necessary to refine our estimate once a better $X_{\mathrm{CO}}$ at the nuclear scale is achieved for NGC 1097.

We then suppose that $M_{\mathrm{H}_{2}}$ and $N_{\mathrm{H}_{2}}$ of NGC 1097 would be a factor of $\gtrsim 2-3$ smaller than those of the molecular torus of NGC 1068 (Imanishi et al. 2016; García-Burillo et al. 2016), which hosts a much more luminous AGN $\left(L_{\mathrm{bol}}=2 \times 10^{45} \mathrm{erg} \mathrm{s}^{-1}\right)$ than NGC 1097 $\left(L_{\mathrm{bol}}=8.6 \times 10^{41} \mathrm{erg} \mathrm{s}^{-1}\right)$. As such, this implies that there is less gas (and likely, optically thinner tori) in LLAGNs than in luminous Seyferts. We would then actually expect less active nuclear star formation and/or inflows in NGC 1097, which will lead to the deficiency of a geometrically thick torus in this LLAGN as compared to luminous AGNs such as NGC 1068 due to the deficiency of the energy source(s): this is an expected trend by the evolutionary torus models $(\S 1)$.

Another remarkable thing is that the envelope of the CND traced by the $\mathrm{CO}(3-2)$ emission and that traced by the NIR $\mathrm{H}_{2}$ emission (Davies et al. 2009; Hicks et al. 2009) are almost identical in NGC 1097 at our available resolutions (Figure 3d). This indicates that the outer radial extent of the molecular CND is unrelated to gas temperature, although the vertical height of the CND could differ (see below), which is consistent with hydrodynamic simulations of CND-scale gas distribution (e.g., Wada et al. 2009). However, we also found that their internal distributions differ significantly: while the two knots stand out in the $\mathrm{CO}(3-2)$ map, the NIR $\mathrm{H}_{2}$ emission is clearly concentrated toward the nucleus, likely reflecting complex excitation conditions including fluorescence around the AGN. Thus, we should be careful to simply assume a linear conversion from NIR $\mathrm{H}_{2}$ line luminosity to CO-based $M_{\mathrm{H}_{2}}$ (Müller Sánchez et al. 2006), when using that luminosity as a tracer of molecular mass.

Gas dynamics is also the key to study the nature of the torus. Figure $4 \mathrm{a}$ and $\mathrm{b}$ show the observed line-of-sight velocity and velocity dispersion maps of the $\mathrm{CO}(3-2)$ line in NGC 1097, respectively. It is evident that rotation (kinematic P.A. $\simeq 118^{\circ}$ ) dominates the global gas dynamics, while streaming motions as well as high velocity dispersions along the gas spirals/arc are detected.

To extract basic beam-deconvolved dynamical information, particularly the rotation velocity $\left(V_{\text {rot }}\right)$ and the velocity dispersion $(\sigma)$, we fitted tilted- 

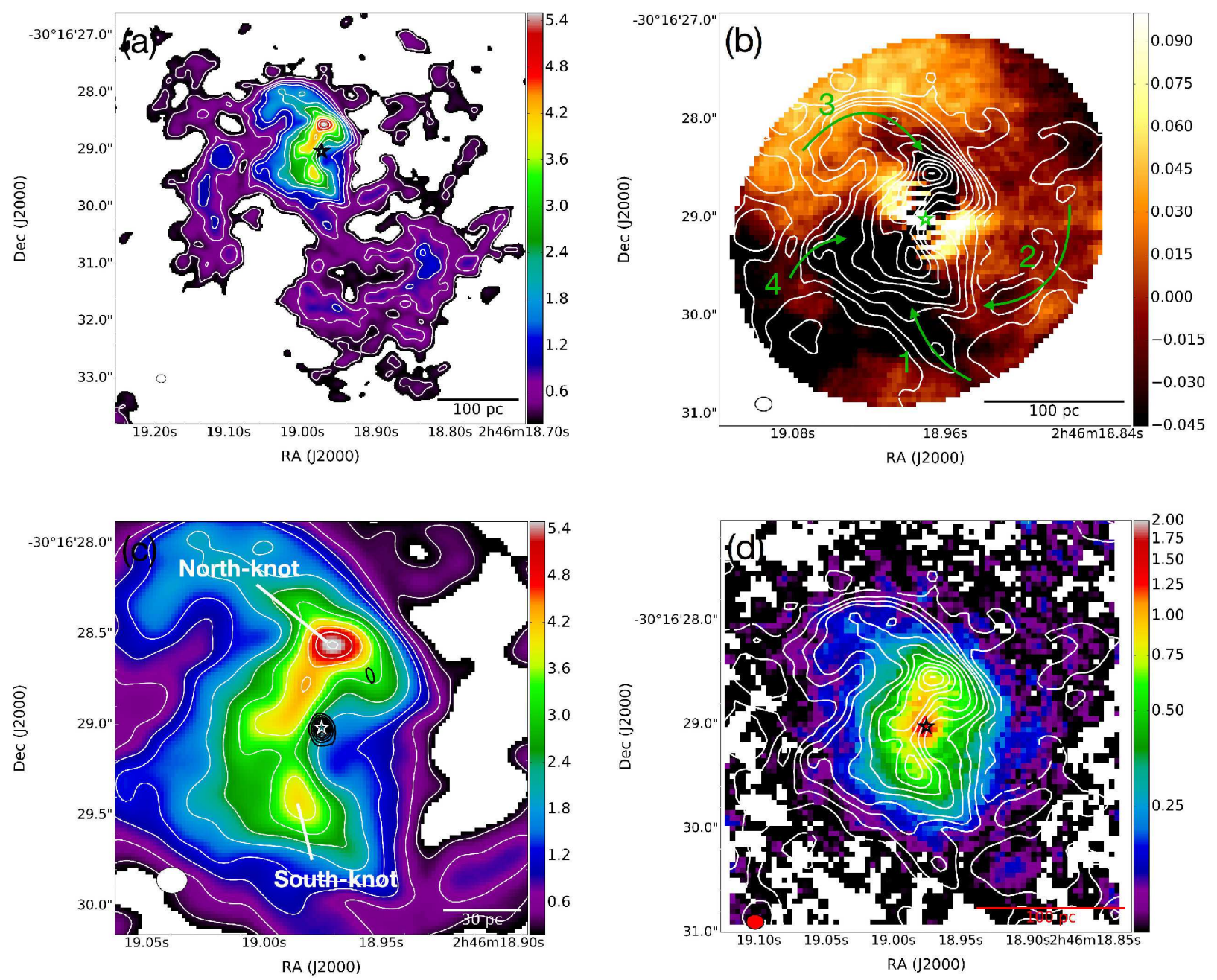

Figure 3. (a) Global spatial distribution of the $\mathrm{CO}(3-2)$ integrated intensity in the central $\sim 500$ pc of NGC 1097 , which mainly consists of the CND and two prominent spiral arms. Inside the CND, an arc-like structure with two (north and south) knots is recognizable. The map shows both the color scale $\left(\mathrm{Jy} \mathrm{beam}^{-1} \mathrm{~km} \mathrm{~s}^{-1}\right.$ unit) and contours $(5,10,15,20,30, \ldots$, and $100 \sigma$, where $1 \sigma=0.062 \mathrm{Jy}_{\text {beam }}^{-1} \mathrm{~km} \mathrm{~s}^{-1}$ ). These contour levels are repeated in the other panels. (b) $\mathrm{CO}(3-2)$ integrated intensity map (contours) overlaid on the $K_{\mathrm{s}}$-band residual (observed distribution - elliptical model) map (arbitrary units; Davies et al. 2009). The two prominent spiral arms (labeled 1 and 3), as well as another two weak candidate arms (labeled 2 and 4 ) are highlighted. (c) A close-up view of (a) at the innermost $\sim 130$ pc. Black contours indicate the $350 \mathrm{GHz}$ continuum emission (Figure 1). (d) $\mathrm{CO}(3-2)$ integrated intensity map (contours) overlaid on the $2.12 \mu \mathrm{m} \mathrm{H}_{2}$ integrated intensity map (in $10^{-17} \mathrm{~W}$ $\mathrm{m}^{-2} \mu \mathrm{m}^{-1}$ units; Davies et al. 2009) at the innermost $\sim 280$ pc. In all panels, the bottom-left ellipse indicates the synthesized beam of the $\operatorname{CO}(3-2)$ map $\left(0^{\prime \prime} .17 \times 0^{\prime \prime} .14\right.$, P.A. $\left.=88^{\circ} .8\right)$, and the central star marks the AGN location.

rings to these observed maps with the ${ }^{3 \mathrm{D}}$ Barolo code (Di Teodoro \& Fraternali 2015). The main parameters are, dynamical center, systemic velocity $\left(V_{\text {sys }}\right), V_{\text {rot }}, \sigma$, galactic inclination $(i)$, and P.A., all of which can be varied for each ring. Our initial fits indicated a dynamical center as the AGN position, $V_{\text {sys }}$ as $1254 \mathrm{~km} \mathrm{~s}^{-1}$. Thus $V_{\text {rot }}, \sigma, i$, and P.A. are free parameters. We modeled 19 concentric rings of $\Delta r=0^{\prime \prime} .05$ from the center. The $V_{\text {rot }}$ and $\sigma$ estimated by the dynamical modeling of the NIR $\mathrm{H}_{2}$ emission (Hicks et al. 2009) were adopted as our initial guesses for the $\mathrm{CO}(3-2)$ modeling. While this code does not account intrinsically for non-circular motions, those can be revealed by subtracting the 2dimensional rotation map from the observed data (e.g., Salak et al. 2016).

We first constructed a model with the full resolution $\mathrm{CO}(3-2)$ cube $\left(0^{\prime \prime} .17 \times 0^{\prime \prime} .11, d V=10 \mathrm{~km} \mathrm{~s}^{-1}\right)$, which we call a MODEL-1. Figure 4c and d respectively show the position velocity diagrams (PVDs) along the global kinematic major and minor axes, overlaid on the observed data. The asymmetric appearances are due to the spatial offset of the AGN from the CO arc. The 

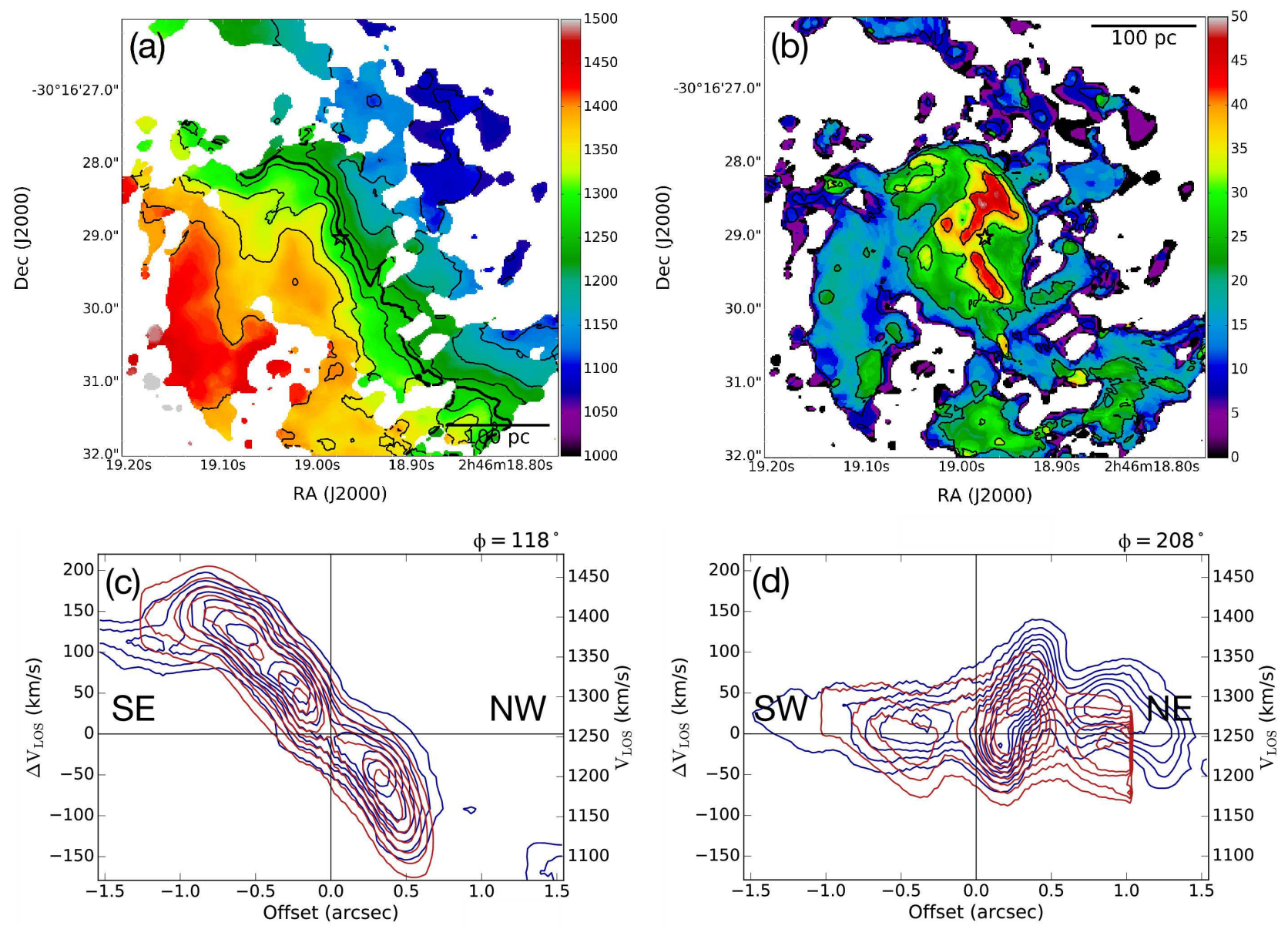

Figure 4. Intensity-weighted (a) mean velocity and (b) velocity dispersion maps of the $\mathrm{CO}(3-2)$ emission in the central 400 pc of NGC 1097. The stars mark the AGN location. The systemic velocity is $1254 \mathrm{~km} \mathrm{~s}^{-1}$ (thick line). Thin contours indicate 1080 to $1400 \mathrm{~km} \mathrm{~s}^{-1}$ in steps of $20 \mathrm{~km} \mathrm{~s}^{-1}$ for (a), and 10 to $40 \mathrm{~km} \mathrm{~s}^{-1}$ in steps of $10 \mathrm{~km} \mathrm{~s}^{-1}$ for (b), respectively. (c)(d) Position-velocity diagrams (PVDs) of the $\mathrm{CO}(3-2)$ line along the adopted major axis $\left(\mathrm{P} . \mathrm{A} .=118^{\circ}\right)$ and minor axis $(\mathrm{P} . \mathrm{A} .=$ $208^{\circ}$ ) shown in the blue contours. The overlaid red contours indicate the PVDs produced by our dynamical model (MODEL-1). Both contours are plotted at $5,10, \ldots, 40 \sigma$, where $1 \sigma=0.84 \mathrm{mJy}_{\text {beam }}{ }^{-1}$. Due to the inclined geometry of the concentric rings, no model component exists at offset $\gtrsim 1^{\prime \prime} .1$ along the minor axis.

global dynamics can be well reproduced by a combination of gas rotation and dispersion, although streaming motions are evident along the minor axis (offset $=+0^{\prime \prime} .3$ to $\left.+1^{\prime \prime} .3\right)$.

The torus itself (offset $=0^{\prime \prime} .0$ ) does not show a significant deviation from this global motion, which is a clear contrast to the case of NGC 1068, where highly perturbed structures are found in the $\mathrm{CO}(6-5)$ velocity field (García-Burillo et al. 2016; Gallimore et al. 2016). This indicates that the molecular torus of NGC 1097 is more quiescent than that of NGC 1068, which is further clarified in the following. Note that no Keplerian rotation was identified in Figure 4c, although the gravitational sphere of influence of the previously inferred $1.2 \times 10^{8}$ $M_{\odot}$ black hole $\left(r \sim 12\right.$ pc or $0^{\prime \prime} .17$; Lewis \& Eracleous 2006) should be resolved at our resolution. The $M_{\mathrm{BH}}$ of NGC 1097 would thus be much smaller than previously thought. This is consistent with the latest estimate $\left(M_{\mathrm{BH}} \sim 10^{6} M_{\odot}\right.$; Hatziminaoglou et al. in prep.) based on a kinematic analysis to $\mathrm{CO}(3-2)$ data also obtained at $\sim 10 \mathrm{pc}$ resolution.

Figure 5a shows the resultant radial profiles of the decomposed $V_{\text {rot }}$ and $\sigma$ of the MODEL-1. In terms of the molecular gas kinematics, the absolute values of $V_{\text {rot }}$ and $\sigma$ agree relatively well $(\lesssim 30 \%)$ with those measured with the $\mathrm{NIR}_{2}$ line (Hicks et al. 2009) at $r \gtrsim 20 \mathrm{pc}$ $\left(0^{\prime \prime} .3\right)$. Although the resultant $\sigma / V_{\text {rot }}$ of the $\mathrm{CO}(3-2)$ line $(\sim 0.2-0.4$; Figure $5 \mathrm{~b})$ is a factor of $\sim 1.5$ smaller than that of the NIR $\mathrm{H}_{2}$ (Hicks et al. 2009) there, this difference would not be so significant as compared to systematic uncertainties of the models according to our experiences. 

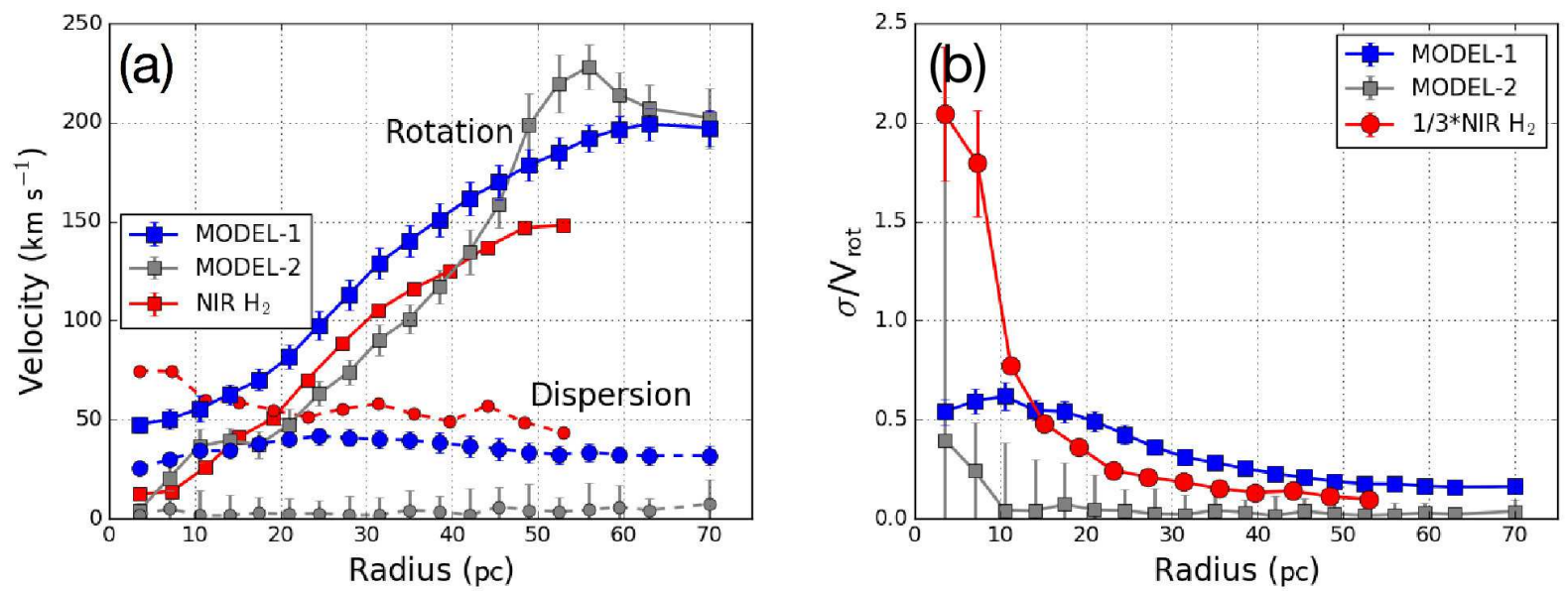

Figure 5. (a) Radial profiles of the rotation velocity ( $V_{\text {rot }}$; squares) and the velocity dispersion ( $\sigma$; circles) of the cold molecular gas estimated from our tilted-ring modelings to the $\mathrm{CO}(3-2)$ velocity field, as well as those from the $\mathrm{NIR} \mathrm{H}_{2}$ velocity field. (b) Radial profiles of the $\sigma / V_{\text {rot }}$ ratio derived from the $\mathrm{CO}(3-2)$ emission line (squares) and the $\mathrm{NIR}_{2} \mathrm{H}_{2}$ emission line (circles; divided by three). In both panels, we plot the data of MODEL-1 and 2 (see $\S 4$ for details). As the MODEL-1 is based on the full resolution $\mathrm{CO}(3-2)$ data, we regard it as fiducial.

On the other hand, the $\mathrm{CO}(3-2)$-based $V_{\text {rot }}$ (MODEL1) departs from that derived from the NIR $\mathrm{H}_{2}$, particularly at the innermost $15 \mathrm{pc}$ (the difference is a factor of $\sim 2-3)$. We suppose this would be due to the mismatched spectral resolutions between our $\mathrm{CO}(3-2)$ cube $\left(10 \mathrm{~km} \mathrm{~s}^{-1}\right)$ and the NIR $\mathrm{H}_{2}$ cube $\left(\sim 70 \mathrm{~km} \mathrm{~s}^{-1}\right.$; Hicks et al. 2009). Indeed, we could achieve a much better agreement $(\lesssim 30 \%)$ in $V_{\text {rot }}$ at $r \lesssim 50$ pc by matching the spectral resolutions (MODEL-2 in Figure 5), while the $\mathrm{CO}(3-2)$-based $\sigma$ now becomes one order of magnitude smaller than that derived from the NIR $\mathrm{H}_{2}$. Now that the velocity width of the $\mathrm{CO}(3-2)$ emission line ( $\sim 100 \mathrm{~km} \mathrm{~s}^{-1}$; Figure 4c) is comparable to the matched spectral resolution $\left(\sim 70 \mathrm{~km} \mathrm{~s}^{-1}\right)$, the $\mathrm{CO}(3-2)$ velocity field can be modelled well by a combination of $V_{\text {rot }}, i$, and P.A., without large $\sigma$. This in turn suggests that the large $\sigma$ returned from the NIR $\mathrm{H}_{2}$ modeling already pointed out a genuine difference in $\sigma$ between the $\mathrm{CO}\left(3^{-}\right.$ 2) and the NIR $\mathrm{H}_{2}$ lines.

In either MODEL, the $\mathrm{CO}(3-2)$-based $\sigma / V_{\text {rot }}$ ratios are much smaller than those derived from the NIR $\mathrm{H}_{2}$ (Figure $5 \mathrm{~b}$ ) at $r \lesssim 20 \mathrm{pc}$. Thus, while the absolute difference of the gas dynamics probed by each tracer is not well constrained at the center of NGC 1097, we would naively imply that cold ( 100 - 300 K in NGC 1097; Izumi et al. 2013) molecular gas is distributed in a thinner layer in and around the torus than the hot $(\sim 1000$
K) component ${ }^{2}$. Regarding the cold molecular torus, an estimated scale height with the value of $\sigma / V_{\text {rot }} \sim 0.65$ (MODEL-1) is then $\sim 4.5 \mathrm{pc}$ at $r=7 \mathrm{pc}$ : we regard this MODEL- 1 as fiducial because it is based on the full resolution $\mathrm{CO}(3-2)$ data.

It is particularly worth noting that the $\sigma / V_{\text {rot }}$ ratio of NGC 1097 at the central $r<10 \mathrm{pc}(\lesssim 0.65$; MODEL1 ) is significantly smaller than that of NGC 1068 (>1; García-Burillo et al. 2016). This is not expected if the disk gravity solely controls the torus thickness, considering the higher $M_{\text {dyn }}$ in NGC 1068 than in NGC 1097 at their very centers $(\S 1)$. Again, the implied less-active star formation and/or inflows from the smaller $M_{\mathrm{H}_{2}}$, as well as the orders of magnitude less-luminous AGN activity in NGC 1097 than those in NGC $1068^{3}$, would contribute to squash the thick torus in this LLAGN (e.g., Vollmer et al. 2008; Wada et al. 2009; Wada 2012). Note that, very recently, Espada et al. (2017) also reported a cold molecular gas deficiency at the heart of Cen A. As the $L_{\text {bol }}$ of Cen A is $\sim 3 \times 10^{42} \mathrm{erg} \mathrm{s}^{-1}$ (LLAGN; Prieto et al. 2010), their findings would also fit the evolutionary torus models.

In summary, although the actual physical process remains unclear, we support the theoretical prediction that geometrically and optically thick tori will gradu-

\footnotetext{
${ }^{2} \mathrm{~A} \sigma / V_{\text {rot }}$ is often used as a proxy of the aspect ratio of a disk under the vertical hydrostatic equilibrium.

3 This AGN may host molecular outflow at the torus region, which would also thicken the scale height (Gallimore et al. 2016; Imanishi et al. 2016).
} 
ally become deficient as AGNs evolve from luminous Seyferts to more quiescent LLAGNs. It is mandatory to enlarge the sample with $\lesssim 10$ pc resolution cold gas measurements to confirm this trend.

We thank the anonymous referee for his/her careful reading and useful comments which greatly improved this paper. This paper makes use of the following ALMA data: ADS/JAO.ALMA\#2015.1.00126.S and \#2012.1.00187.S. ALMA is a partnership of ESO (representing its member states), NSF (USA) and NINS
(Japan), together with NRC (Canada), NSC and ASIAA (Taiwan), and KASI (Republic of Korea), in cooperation with the Republic of Chile. The Joint ALMA Observatory is operated by ESO, AUI/NRAO and NAOJ. We sincerely thank E. K. S. Hicks for kindly providing the NIR $\mathrm{H}_{2}$ kinematic data. T.I., N.K., K.W., and K.K. are supported by JSPS KAKENHI Grant Number 17K14247, 16K17670, 16H03959, and 25247019, respectively. T.I. was supported by the ALMA Japan Research Grant of NAOJ Chile Observatory, NAOJ-ALMA-170.

\section{REFERENCES}

Aalto, S., Muller, S., Costagliola, F., et al. 2017, ArXiv e-prints, arXiv:1702.05458

Antonucci, R. 1993, ARA\&A, 31, 473

Bolatto, A. D., Wolfire, M., \& Leroy, A. K. 2013, ARA\&A, 51,207

Burtscher, L., Meisenheimer, K., Tristram, K. R. W., et al. 2013, A\&A, 558, A149

Davies, R. I., Maciejewski, W., Hicks, E. K. S., et al. 2009, ApJ, 702, 114

Di Teodoro, E. M., \& Fraternali, F. 2015, MNRAS, 451, 3021

Elitzur, M., \& Shlosman, I. 2006, ApJL, 648, L101

Espada, D., Matsushita, S., Miura, R. E., et al. 2017, ArXiv e-prints, arXiv:1706.05762

Fathi, K., Storchi-Bergmann, T., Riffel, R. A., et al. 2006, ApJL, 641, L25

Fathi, K., Lundgren, A. A., Kohno, K., et al. 2013, ApJL, 770, L27

Gallimore, J. F., Elitzur, M., Maiolino, R., et al. 2016, ApJL, 829, L7

García-Burillo, S., Combes, F., Usero, A., et al. 2014, A\&A, 567, A125

García-Burillo, S., Combes, F., Ramos Almeida, C., et al. 2016, ApJL, 823, L12

González-Martín, O., Masegosa, J., Márquez, I., et al. 2015, A\&A, 578, A74

González-Martín, O., Masegosa, J., Hernán-Caballero, A., et al. 2017, ApJ, 841, 37

Greenhill, L. J., Gwinn, C. R., Antonucci, R., \& Barvainis, R. 1996, ApJL, 472, L21

Hicks, E. K. S., Davies, R. I., Malkan, M. A., et al. 2009, ApJ, 696, 448

Ho, L. C. 2008, ARA\&A, 46, 475

Hsieh, P.-Y., Ho, P. T. P., Kohno, K., Hwang, C.-Y., \& Matsushita, S. 2012, ApJ, 747, 90
Imanishi, M., Nakanishi, K., \& Izumi, T. 2016, ApJL, 822, L10

Izumi, T., Kohno, K., Martín, S., et al. 2013, PASJ, 65, 100

Jaffe, W., Meisenheimer, K., Röttgering, H. J. A., et al. 2004, Nature, 429, 47

Klaas, U., Haas, M., Müller, S. A. H., et al. 2001, A\&A, 379,823

Lewis, K. T., \& Eracleous, M. 2006, ApJ, 642, 711

Lou, Y.-Q., Yuan, C., Fan, Z., \& Leon, S. 2001, ApJL, 553, L35

Marinucci, A., Bianchi, S., Matt, G., et al. 2016, MNRAS, 456, L94

McMullin, J. P. and Waters, B. and Schiebel, D. and Young, W. and Golap, K. 2007, in Astronomical Society of the Pacific Conference Series, Vol. 376, Astronomical Data Analysis Software and Systems XVI, ed. R. A.

Shaw, F. Hill, \& D. J. Bell, 127

Müller Sánchez, F., Davies, R. I., Eisenhauer, F., et al. 2006, A\&A, 454, 481

Müller-Sánchez, F., Prieto, M. A., Mezcua, M., et al. 2013, ApJL, 763, L1

Nemmen, R. S., Storchi-Bergmann, T., Yuan, F., et al. 2006, ApJ, 643, 652

Nomura, M., Ohsuga, K., Takahashi, H. R., Wada, K., \& Yoshida, T. 2016, PASJ, 68, 16

Orienti, M., \& Prieto, M. A. 2010, MNRAS, 401, 2599

Piñol-Ferrer, N., Fathi, K., Lundgren, A., \& van de Ven, G. 2011, MNRAS, 414, 529

Prieto, M. A., Maciejewski, W., \& Reunanen, J. 2005, AJ, 130,1472

Prieto, M. A., Reunanen, J., Tristram, K. R. W., et al. 2010, MNRAS, 402, 724

Quillen, A. C., Frogel, J. A., Kuchinski, L. E., \& Terndrup, D. M. 1995, AJ, 110, 156

Salak, D., Nakai, N., Hatakeyama, T., \& Miyamoto, Y. 2016, ApJ, 823, 68 
Sault, R. J. and Teuben, P. J. and Wright, M. C. H. 1995, in Astronomical Society of the Pacific Conference Series, Vol. 77, Astronomical Data Analysis Software and Systems IV, ed. R. A. Shaw, H. E. Payne, \& J. J. E. Hayes, 433

Storchi-Bergmann, T., Baldwin, J. A., \& Wilson, A. S. 1993, ApJL, 410, L11

Storchi-Bergmann, T., Nemmen, R. S., Spinelli, P. F., et al. 2005, ApJL, 624, L13
Tully, R. B. 1988, Nearby Galaxies Catalog (Cambridge Univ. Press, Cambridge)

van de Ven, G., \& Fathi, K. 2009, ApJ, 723, 767

Vollmer, B., Beckert, T., \& Davies, R. I. 2008, A\&A, 491, 441

Wada, K. 2012, ApJ, 758, 66

Wada, K., Papadopoulos, P. P., \& Spaans, M. 2009, ApJ, 702,63 